\title{
Risk Assessment of Passive Smoking Based on Analysis of Hair Nicotine and Cotinine as Exposure Biomarkers by In-Tube Solid-Phase Microextraction Coupled On-Line to LC-MS/MS
}

\author{
Hiroyuki Kataoka *(D), Sanae Kaji and Maki Moai
}

check for

updates

Citation: Kataoka, H.; Kaji, S.; Moai, M. Risk Assessment of Passive

Smoking Based on Analysis of Hair Nicotine and Cotinine as Exposure Biomarkers by In-Tube Solid-Phase Microextraction Coupled On-Line to LC-MS/MS. Molecules 2021, 26, 7356. https://doi.org/10.3390/molecules 26237356

Academic Editor: Joselito P. Quirino

Received: 19 November 2021

Accepted: 1 December 2021

Published: 3 December 2021

Publisher's Note: MDPI stays neutral with regard to jurisdictional claims in published maps and institutional affiliations.

Copyright: (c) 2021 by the authors. Licensee MDPI, Basel, Switzerland. This article is an open access article distributed under the terms and conditions of the Creative Commons Attribution (CC BY) license (https:/ / creativecommons.org/licenses/by/ $4.0 /)$.
School of Pharmacy, Shujitsu University, Nishigawara, Okayama 703-8516, Japan; 16kj5bo6f-tdxu5@docomo.ne.jp (S.K.); s1914121tc@shujitsu.jp (M.M.)

* Correspondence: hkataoka@shujitsu.ac.jp

\begin{abstract}
Passive smoking due to environmental tobacco smoke is a serious public health concern because it increases the risk of lung cancer and cardiovascular disease. However, the current status and effect of passive smoking in various lifestyles are not fully understood. In this study, we measured hair nicotine and cotinine levels as exposure biomarkers in non-smokers and assessed the risk from the actual situation of passive smoking in different lifestyle environments. Nicotine and cotinine contents in hair samples of 110 non-smoker subjects were measured by in-tube solid-phase microextraction with on-line coupling to liquid chromatography-tandem mass spectrometry, and self-reported lifestyle questionnaires were completed by the subjects. Nicotine and cotinine were detected at concentrations of $1.38 \mathrm{ng} \mathrm{mg}^{-1}$ and $12.8 \mathrm{pg} \mathrm{mg}^{-1}$ respectively in the hair of non-smokers, with levels significantly higher in subjects who reported being sensitive to tobacco smoke exposure. These levels were also affected by type of food intake and cooking method. Nicotine and cotinine in hair are useful biomarkers for assessing the effects of passive smoking on long-term exposure to environmental tobacco smoke, and our analytical methods can measure these exposure levels in people who are unaware of passive smoking. The results of this study suggest that the environment and places of tobacco smoke exposure and the lifestyle behaviors therein are important for the health effects of passive smoking.
\end{abstract}

Keywords: passive smoking; nicotine; cotinine; in-tube solid-phase microextraction (SPME); liquid chromatography-tandem mass spectrometry (LC-MS/MS); risk assessment

\section{Introduction}

Tobacco smoking and exposure to environmental tobacco smoke are considered risk factors for cancers, cardiovascular diseases and respiratory disease, and the health effects of tobacco smoke have become a serious social problem [1-3]. According to a 2020 World Health Organization (WHO) survey, more than 8 million deaths worldwide are caused by tobacco each year, and more than 7 million of those attributed to direct tobacco use and around 1.2 million to indirect exposure through passive smoking [4]. In addition, $40 \%$ of children (under 14 years old), 33\% of non-smoking men and 35\% of non-smoking women are indirectly exposed to tobacco smoke, of whom 166,000 children, 156,000 men and 281,000 women die each year from passive smoking [5,6]. Furthermore, persons with passive smoking have been reported to have a 1.3-fold higher risk of developing lung cancer than those without passive smoking [7]. In addition, wives of husbands who smoke are reported to have twice the risk of developing lung cancer compared to wives of husbands who do not smoke [8]. Therefore, environmental tobacco smoke, including second-hand smoke such as sidestream smoke from cigarettes and exhaled smoke by smokers, and third-hand smoke from clothing, curtains and indoor wallpaper, may be an important risk factor for lung cancer and tobacco-related diseases in non-smokers [6,9]. 
Passive smoking not only increases the risk of cancer [9-15] but has also been reported to be associated with the development of cardiovascular disease [16-23], diabetes [6,24,25], metabolic syndrome [26], psychiatric disorders (depression) [27] and cognitive decline [28]. This is a serious problem associated with a variety of adverse health effects, especially for non-smoking children and pregnant women [29,30]. For example, health hazards caused by passive smoking in children are more severe than in adults, bringing increased risk of growth retardation [31,32] and sudden infant death syndrome (SIDS) [33,34] in fetuses born to mothers exposed to tobacco smoke. In addition, passive smoking in infancy has been linked to obesity, diabetes and metabolic syndrome in adulthood [35,36]. Since passive smoking is unintentional and unavoidable in the presence of smokers in the close relatives, there is an urgent need for effective measures to reduce passive smoking worldwide from both public health and clinical aspects [29]. Therefore, it is essential to determine the facts of biological exposure to prevent health hazards caused by passive smoking [1,3,37], however, the current status and effects of passive smoking in various lifestyle behaviors in different environments are not fully understood.

Tobacco-related compounds such as nicotine, its metabolite cotinine and tobaccospecific nitrosamines have been used as biomarkers of exposure to tobacco smoke [6]. However, their half-lives in urine and blood range from a few hours to 3-4 days, and these compounds are rapidly eliminated after exposure to tobacco smoke [6]. In addition, their concentrations in these matrices are much lower in passive smokers than in active smokers, making them unsuitable for assessing the effects of long-term exposure to environmental tobacco smoke in non-smokers. In contrast, hair samples have been frequently used to assess and monitor the bioaccumulation due to long-term exposure to drugs, environmental pollutants, etc. [38-41], as the amounts of these compounds in the hair are less affected by daily exposure and variations in metabolism than those in other biological matrices [6,42]. Furthermore, hair samples can be collected easily and less invasively and can be stored at room temperature for up to five years $[6,43]$. Recently, we developed a simple and sensitive method for the determination of nicotine and cotinine $[44,45]$ and tobacco-specific nitrosamines [46] in hair by in-tube solid-phase microextraction (SPME) with on-line coupling to liquid chromatography-tandem mass spectrometry (LC-MS/MS) [47-49] and assessed differences in levels of these compounds in active and passive smokers. In the present study, we measured hair nicotine and cotinine levels in healthy non-smokers and asked self-reported lifestyle questions to assess the risk from the actual status of passive smoking in different lifestyle environments.

\section{Results}

The automated on-line in-tube SPME/LC-MS/MS method [45] is sufficiently selective, sensitive and precise for nicotine and cotinine analysis (Table S1). This method was successfully applied to the analysis of nicotine and cotinine at pg levels per $1 \mathrm{mg}$ of hair samples without any interference peaks. Typical chromatograms obtained from $200 \mathrm{pg} \mathrm{mL}^{-1}$ standard solution and a sample corresponding to $0.2 \mathrm{mg}$ of hair were shown in Figure 1. In this study, the analysis method is the same as in the previous paper [45], but the number of non-smokers was increased from 58 to 110 , and a questionnaire on lifestyle was administered to analyze the relationship with the exposure level. In a previous study [45], the levels of nicotine and cotinine in the hair of eight smokers were $43.12 \mathrm{ng} \mathrm{mg}^{-1}$ and $655 \mathrm{pg} \mathrm{mg}^{-1}$, respectively, and those of 20 non-smokers (not even passive smokers) were $1.86 \mathrm{ng} / \mathrm{mg}$ and $8 \mathrm{pg} \mathrm{mg}^{-1}$, respectively. The levels of nicotine and cotinine were 23 and 82 times higher in smokers than in non-smokers, respectively. In the present study, the contents of nicotine and cotinine in the hair of 110 non-smokers differ by more than 100-fold in concentration, and were $1.38 \pm 1.36 \mathrm{ng} \mathrm{mg}^{-1}$ and $12.8 \pm 13.7 \mathrm{pg} \mathrm{mg}^{-1}$, respectively. In particular, the levels of hair nicotine and cotinine in 46 non-smokers (not even passive smokers) were $1.11 \mathrm{ng} \mathrm{mg}^{-1}$ and $8 \mathrm{pg} \mathrm{mg}^{-1}$, respectively, similar to the previous results. As shown in Table 1 (Question 1 and 2), however, there was no significant difference in these contents by sex and age of participants. 
(A) Standard solution (200 $\mathrm{pg} / \mathrm{mL})$
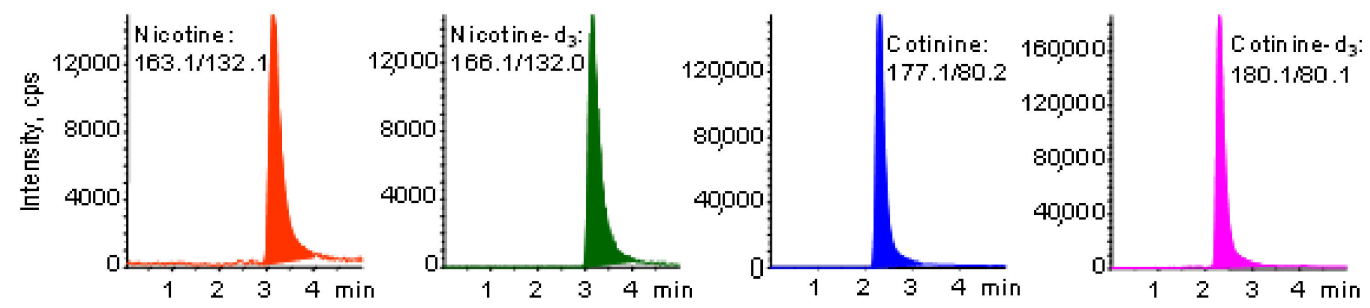

(B) Non smoker (0.2 mg hair)
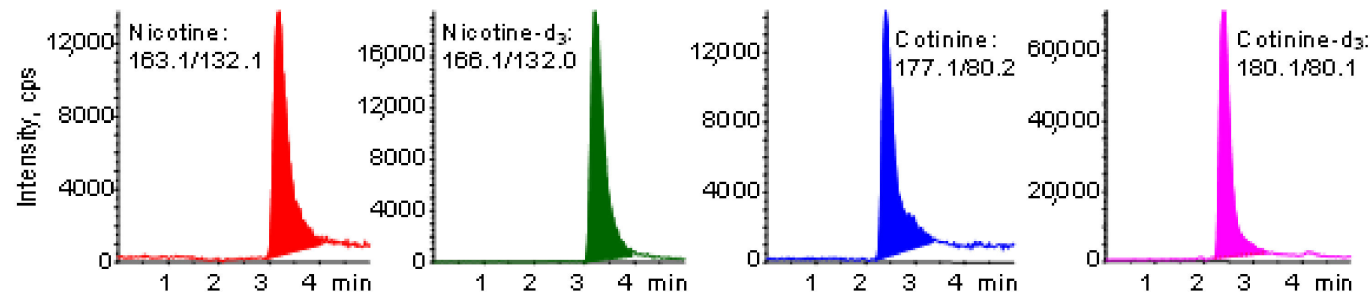

Figure 1. Typical MRM chromatograms obtained from (A) standard solution and (B) hair sample of non-smoker by in-tube SPME LC-MS/MS. Analytical conditions are described in the Experimental section.

Table 1. Nicotine and cotinine contents in non-smoker's hair based on lifestyle questionnaires.

\begin{tabular}{|c|c|c|c|}
\hline \multirow{2}{*}{ Question/Answer } & \multirow{2}{*}{$n$} & \multicolumn{2}{|c|}{ Content in Hair/Mean (Min. Max.) } \\
\hline & & Nicotine (ng mg ${ }^{-1}$ ) & Cotinine $\left(\mathrm{pg} \mathrm{mg}^{-1}\right)$ \\
\hline \multicolumn{4}{|l|}{ 1. Sex } \\
\hline Male & 30 & $1.63(0.20 \sim 7.72)$ & $14.4(4.8 \sim 43.9)$ \\
\hline Female & 80 & $1.29(0.07 \sim 4.99)$ & $12.2(2.7 \sim 99.1)$ \\
\hline $\mathrm{P}^{1}$ (Male/female) & & 0.120 & 0.234 \\
\hline \multicolumn{4}{|l|}{ 2. Age } \\
\hline 29 years old and under & 79 & $1.39(0.07 \sim 5.40)$ & $12.1(2.7 \sim 99.1)$ \\
\hline 30 years old and over & 31 & $1.37(0.17 \sim 7.72)$ & $14.6(4.3 \sim 59.3)$ \\
\hline P (Younger/older) & & 0.477 & 0.198 \\
\hline \multicolumn{4}{|l|}{ 3. Daily sleeping time } \\
\hline Less than $6 \mathrm{~h}$ & 30 & $1.19(0.07 \sim 3.89)$ & $10.8(4.1 \sim 59.3)$ \\
\hline More than $6 \mathrm{~h}$ & 80 & $1.45(0.10 \sim 37.72)$ & $13.6(2.7 \sim 99.1)$ \\
\hline P (Shorter/longer) & & 0.188 & 0.171 \\
\hline \multicolumn{4}{|l|}{ 4. Stress awareness } \\
\hline Sometimes $^{2}$ & 68 & $1.39(0.07 \sim 7.72)$ & $13.8(2.7 \sim 99.1)$ \\
\hline Frequently ${ }^{2}$ & 42 & $1.38(0.17 \sim 4.99)$ & $11.2(4.4 \sim 59.3)$ \\
\hline P (Sometimes/frequently) & & 0.483 & 0.169 \\
\hline \multicolumn{4}{|c|}{ 5. Passive smoking awareness } \\
\hline Never & 46 & $1.11(0.07 \sim 4.55)$ & $8.1(2.7 \sim 21.0)$ \\
\hline Sometimes & 55 & $1.52(0.16 \sim 7.72)$ & $13.8(3.8 \sim 59.3)$ \\
\hline Frequently & 9 & $1.94(0.72 \sim 3.60)$ & $31.0(6.6 \sim 99.1)$ \\
\hline P (Never/sometimes) & & 0.064 & 0.001 \\
\hline P (Never/frequently) & & 0.023 & 0.00001 \\
\hline \multicolumn{4}{|l|}{ 6. Exposure to other smoke } \\
\hline Never & 55 & $1.35(0.12 \sim 5.40)$ & $14.0(2.7 \sim 99.1)$ \\
\hline Sometimes Always & 55 & $1.42(0.07 \sim 7.72)$ & $11.6(3.8 \sim 47.0)$ \\
\hline $\mathrm{P}$ (Never/yes) & & 0.397 & 0.177 \\
\hline \multicolumn{4}{|l|}{ 7. Frequency of tea drinking } \\
\hline Sometimes/Frequently & 46 & $1.49(0.17 \sim 7.72)$ & $12.4(4.0 \sim 59.3)$ \\
\hline Always & 64 & $1.30(0.07 \sim 5.40)$ & $13.1(2.7 \sim 99.1)$ \\
\hline $\mathrm{P}$ (Less/more) & & 0.241 & 0.409 \\
\hline
\end{tabular}


Table 1. Cont.

\begin{tabular}{|c|c|c|c|}
\hline \multirow{2}{*}{ Question/Answer } & \multirow{2}{*}{$n$} & \multicolumn{2}{|c|}{ Content in Hair/Mean (Min. Max.) } \\
\hline & & Nicotine (ng mg ${ }^{-1}$ ) & Cotinine (pg mg ${ }^{-1}$ ) \\
\hline \multicolumn{4}{|l|}{ 8. Fat and fatty food intake } \\
\hline Sometimes & 8 & $1.82(0.37 \sim 7.72)$ & $12.7(5.1 \sim 35.2)$ \\
\hline Frequently & 81 & $1.27(0.07 \sim 4.99)$ & $11.8(2.7 \sim 99.1)$ \\
\hline Always & 21 & $1.64(0.20 \sim 5.40)$ & $16.9(3.8 \sim 47.0)$ \\
\hline P (Frequently/always) & & 0.405 & 0.227 \\
\hline \multicolumn{4}{|l|}{ 9. Vegetable intake } \\
\hline Sometimes & 31 & $1.27(0.10 \sim 4.54)$ & $9.7(2.7 \sim 32.5)$ \\
\hline Frequently & 64 & $1.36(0.07 \sim 7.72)$ & $15.1(4.0 \sim 99.1)$ \\
\hline Always & 15 & $1.71(0.20 \sim 4.99)$ & $9.4(3.8 \sim 22.0)$ \\
\hline P (Sometimes/always) & & 0.135 & 0.450 \\
\hline \multicolumn{4}{|l|}{ 10. Consumption of vegetables } \\
\hline Raw & 55 & $1.29(0.07 \sim 7.72)$ & $12.0(2.7 \sim 99.1)$ \\
\hline Boiled & 21 & $1.30(0.12 \sim 3.60)$ & $13.0(4.3 \sim 59.3)$ \\
\hline Pan-fried & 34 & $1.57(0.10 \sim 5.42)$ & $14.0(3.8 \sim 67.0)$ \\
\hline P (Raw / pan-fried $)$ & & 0.232 & 0.390 \\
\hline P (Boiled/pan-fried) & & 0.192 & 0.249 \\
\hline \multicolumn{4}{|l|}{ 11. Spice use } \\
\hline Sometimes & 36 & $1.54(0.12 \sim 5.40)$ & $12.7(4.0 \sim 59.3)$ \\
\hline Frequently & 58 & $1.41(0.10 \sim 7.72)$ & $13.7(2.7 \sim 99.1)$ \\
\hline Always & 16 & $0.93(0.07 \sim 3.48)$ & $9.7(4.9 \sim 31.7)$ \\
\hline P (Sometimes/always) & & 0.055 & 0.169 \\
\hline \multicolumn{4}{|l|}{ 12. Meat intake } \\
\hline Sometimes & 14 & $1.20(0.28 \sim 4.99)$ & $10.5(5.1 \sim 31.7)$ \\
\hline Frequently & 67 & 1.47 (0.07 7.72) & $13.5(2.7 \sim 99.1)$ \\
\hline Always & 29 & $1.28(0.12 \sim 3.60)$ & $12.2(4.8 \sim 67.0)$ \\
\hline P (Sometimes/always) & & 0.414 & 0.311 \\
\hline \multicolumn{4}{|l|}{ 13. Consumption of meat } \\
\hline Boiled & 9 & $0.81(0.30 \sim 2.19)$ & $7.6(5.1 \sim 13.7)$ \\
\hline Pan-fried/Deep-fried & 28 & $1.02(0.10 \sim 3.25)$ & $9.6(2.7 \sim 31.7)$ \\
\hline Grilled & 73 & 1.59 (0.07 7.72) & $19.1(4.0 \sim 99.1)$ \\
\hline P (Boiled/grilled) & & 0.071 & 0.096 \\
\hline P (Fried/grilled) & & 0.034 & 0.053 \\
\hline \multicolumn{4}{|l|}{ 14. Seafood intake } \\
\hline Sometimes & 48 & $1.40(0.12 \sim 5.40)$ & $12.0(2.7 \sim 47.0)$ \\
\hline Frequently & 54 & 1.37 (0.07 7.72) & $14.1(3.8 \sim 99.1)$ \\
\hline Always & 8 & $1.24(0.17 \sim 3.44)$ & $8.9(5.6 \sim 1.45)$ \\
\hline $\mathrm{P}$ (Sometimes/always) & & 0.478 & 0.205 \\
\hline \multicolumn{4}{|l|}{ 15. Consumption of seafood } \\
\hline Raw & 21 & $1.12(0.12 \sim 4.99)$ & $17.9(4.4 \sim 99.1)$ \\
\hline Boiled & 33 & $1.38(0.29 \sim 5.40)$ & $12.6(4.0 \sim 43.9)$ \\
\hline Grilled & 56 & $1.48(0.07 \sim 7.72)$ & $11.0(2.7 \sim 67.0)$ \\
\hline P (Raw / grilled) & & 0.155 & 0.036 \\
\hline P (Boiled/grilled) & & 0.369 & 0.228 \\
\hline
\end{tabular}

${ }^{1}$ Probability (significant difference T-test). ${ }^{2}$ Sometimes: 3 days and under per week; frequently: 4 days and over per week.

The nicotine and cotinine contents obtained from hair analyses of 110 non-smokers were compared with data of reported lifestyle factors, such as daily sleeping time, stress, passive smoking, food consumption frequency and type. As shown in Table 1, the levels of nicotine or cotinine in hair were not affected by sleeping time or degree of stress. In this table, "Sometimes" means three days and under per week and "Frequently" means four days and over per week. In the questionnaire survey of passive smoking frequency among 110 non-smokers, 46 reported never, 55 sometimes and 9 frequent or always. Their hair nicotine contents were $1.11 \pm 1.11,1.52 \pm 1.54$ and $1.94 \pm 1.18 \mathrm{ng} \mathrm{mg}^{-1}$, and hair 
cotinine contents were $8.1 \pm 4.2,13.8 \pm 11.8$ and $31.0 \pm 31.9 \mathrm{pg} \mathrm{mg}^{-1}$, respectively. Both compounds were detected in the hair of non-smokers who reported never being exposed to environmental tobacco smoke, but at significantly lower concentrations than in those who reported exposure. Whisker-box plots displaying the medians and interquartile ranges of hair nicotine and cotinine are presented in Figure 2A,B. These results indicate that the greater the awareness of passive smoking, the greater the accumulation of both compounds in the hair. Furthermore, the levels of nicotine and cotinine in hair were not affected by the presence or absence of exposure to non-cigarette smoke, such as cooking or wood burning, indicating that passive smoking could be detected selectively by hair analysis.
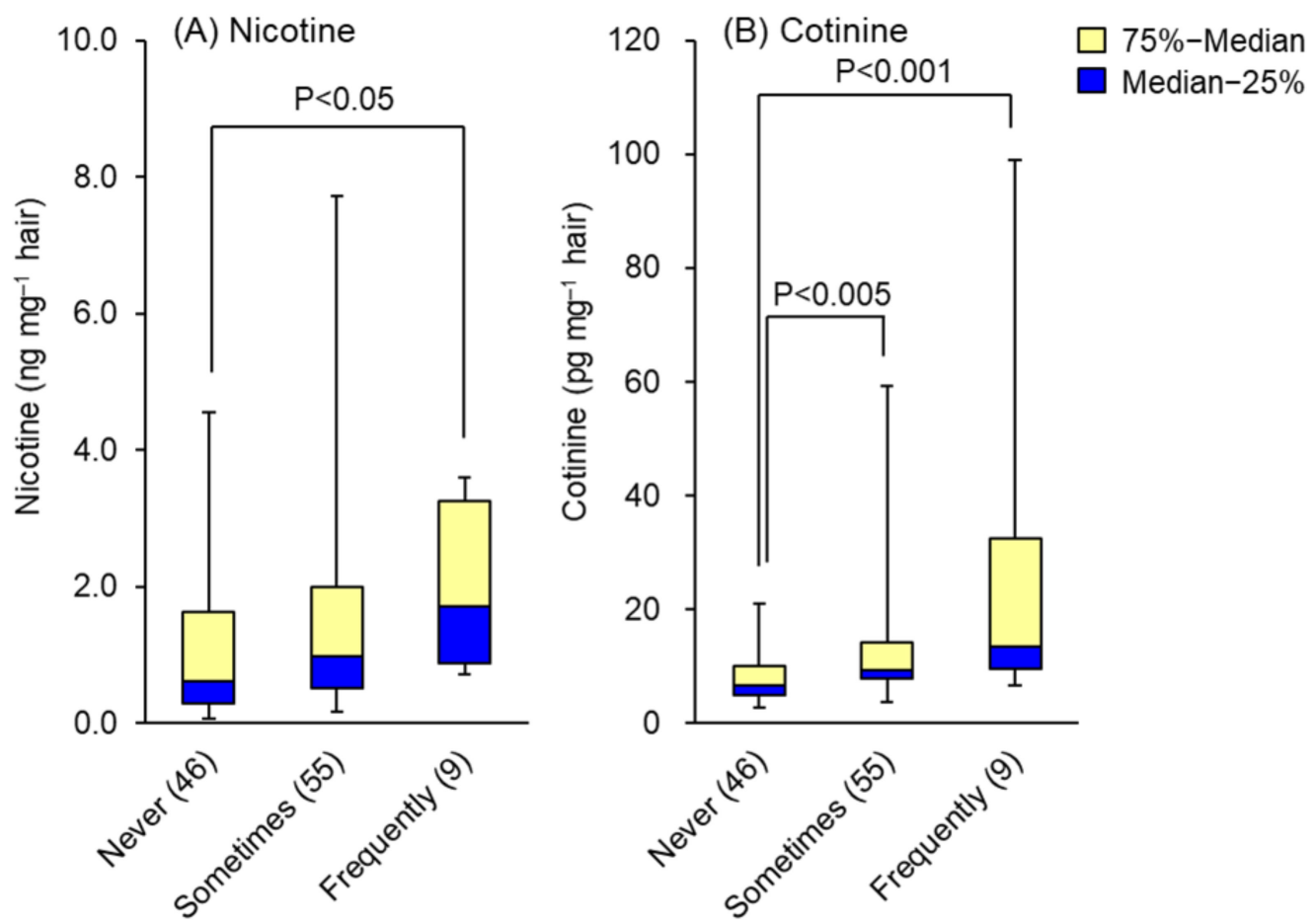

Figure 2. Effects of passive smoking awareness on the levels of $(\mathbf{A})$ nicotine and $(\mathbf{B})$ cotinine in hair. Data are presented as whisker-box plots displaying medians and interquartile ranges. Not at all: no awareness of passive smoking at all; sometimes: 3 days and under per week; frequently: for 4 days and over per week. The number of subjects is shown in parentheses. Top of each box, 75th percentile; bottom of each box, 25th percentile; solid center line, 50th percentile; error bars, non-outlier range.

There were no significant differences in hair nicotine and cotinine levels between frequencies of food intake, but those who consumed more spices tended to have lower levels. On the other hand, there were no significant differences in hair nicotine and cotinine levels depending on the frequency of meat consumption, but these levels tended to increase in those who ate grilled meat more often. Hair nicotine contents by consumption of boiled (9), fried (28) and grilled (73) meat were $0.81 \pm 0.59,1.02 \pm 0.80$ and $1.59 \pm 1.55 \mathrm{ng} \mathrm{mg}^{-1}$, and hair cotinine contents were $8.6 \pm 2.7,9.6 \pm 6.3$ and $19.1 \pm 20.5 \mathrm{pg} \mathrm{mg}^{-1}$, respectively. Whisker-box plots of medians and interquartile ranges of hair nicotine and cotinine are shown in Figure 3A,B. These results may be affected by the tobacco smoke environment in places where grilled meat is eaten, such as grilled meat restaurants, since there was almost no effect of nicotine and cotinine from smoke other than tobacco smoke, such as smoke generated by cooking. 

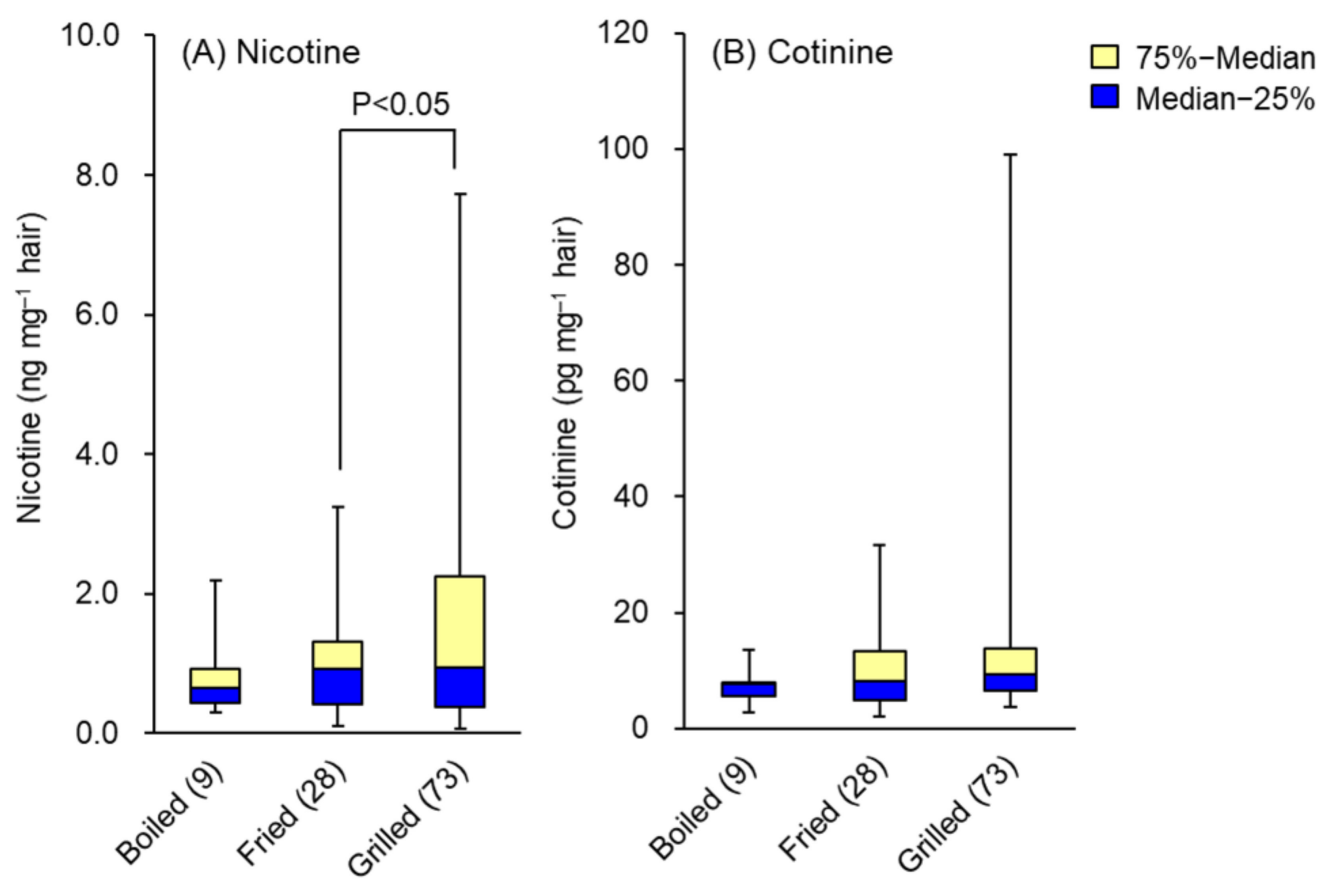

Figure 3. Effects of cooking method of meat on the levels of (A) nicotine and (B) cotinine in hair. Data are presented as whisker-box plots displaying medians and interquartile ranges. The number of subjects is shown in parentheses. Top of each box, 75th percentile; bottom of each box, 25th percentile; solid center line, 50th percentile; error bars, non-outlier range.

\section{Discussion}

Using the on-line in-tube SPME/LC-MS/MS method, the effects of tobacco smoke exposure can be assessed selectively and sensitively by analyzing as little as $1 \mathrm{mg}$ of hair. Concentrations of nicotine and cotinine in hair reflect long-term exposure to tobacco smoke and are useful to assess the health effects of environmental tobacco smoke even in people unaware of passive smoking. Although cotinine concentrations in the hair of non-smokers were much lower than nicotine concentrations, there was a marked difference in cotinine concentrations between those who were aware of passive smoking and those who were not. This means that cotinine is more reflective of the reality of passive smoking than nicotine.

The consumption of food affected the accumulation of nicotine and cotinine in the hair. Those who consumed spices more frequently had lower levels of nicotine and cotinine in their hair, suggesting that spices help to remove tobacco compounds from the body. This may be related to the preventive effect of spices such as curcumin on lung cancer [50]. As nicotine and cotinine levels in hair are not affected by cooking smoke, high levels of nicotine and cotinine in the hair of non-smokers who eat grilled meat more frequently suggest that they may be affected by passive smoking in places such as grill restaurants and barbecue. Indeed, in Japan, smoking is relatively common in environments where groups of people drink alcohol and eat grilled meat. People unaware of passive smoking may have been unconsciously exposed to low concentrations of environmental tobacco smoke over long periods of time since nicotine and cotinine were detected in their hair. Therefore, it is important to understand the actual situation of exposure to environmental tobacco smoke because it is impossible to know when and where one will be exposed to passive smoking unless smoking itself is banned, and non-smokers may be forced to smoke passively for long periods of time in a variety of environments. In the future, in order to avoid the risk of passive smoking, it may be possible to prevent health problems caused by passive smoking not only by avoiding exposure to tobacco smoke, but also by improving eating habits and lifestyle habits. 


\section{Materials and Methods}

\subsection{Reagents and Standard Solutions}

Nicotine and cotinine were purchased from Sigma-Aldrich Japan (Tokyo, Japan). Nicotine- $\mathrm{d}_{3}$ (isotopic purity $98.4 \%$ ), and cotinine- $\mathrm{d}_{3}$ (isotopic purity $99.9 \%$ ) purchased from Toronto Research Chemicals Inc. (Toronto, Ontario, Canada) were used as an internal standard (IS). Stock solutions of $1 \mathrm{mg} \mathrm{mL}^{-1}$ of each compound were prepared by dissolving in methanol. The working solutions were prepared by diluting these stock solutions with distilled water to the required concentration. These prepared solutions were stored at $4{ }^{\circ} \mathrm{C}$ in refrigerator until use. Methanol and distilled water were of LC-MS grade, while all other chemicals were of analytical reagent grade.

\subsection{On-Line Automated Analysis System and Analytical Conditions}

Nicotine and cotinine were measured using an on-line automated analysis system comprising in-tube SPME coupled with LC-MS/MS equipped with a Model 1100 series LC (Agilent Technologies, Böblingen, Germany) and an API 4000 triple quadruple mass spectrometer (Applied Biosystems, Foster City, CA, USA) by the previously reported method [45]. LC-MS/MS data were analyzed using Analyst Software 1.3.1 (Applied Biosystems). An outline of the system is shown in Figure S1 (refer to Supplementary Materials).

A Carboxen 1006 PLOT capillary column (Carboxen molecularsives, film thickness $17 \mu \mathrm{m}, 60 \mathrm{~cm} \times 0.32 \mathrm{~mm}$ i.d., Supelco, Bellefonte, PA, USA) was used as an in-tube SPME device and was connected between the injection needle and the injection loop of the LC autosampler. All controls for extraction, desorption and injection were programmed by the autosampler software (Table S2, Supplementary Materials).

A Polar-RP 80A column $(50 \mathrm{~mm} \times 2.0 \mathrm{~mm}$ i.d., particle size $4 \mu \mathrm{m}$, Phenomenex; Torrance, CA, USA) was used for LC separation at a column temperature of $30^{\circ} \mathrm{C}$, with $2.5 \mathrm{mM}$ ammonium formate/methanol $(25 / 75, v / v)$ as the mobile phase, at a flow rate of $0.2 \mathrm{~mL} \mathrm{~min}^{-1}$ [45]. Electro spray ionization (ESI)-MS/MS of nicotine, cotinine and their stable isotope-labeled compounds was performed in positive ion mode at $4000 \mathrm{~V}$ and $600{ }^{\circ} \mathrm{C}$ by multiple reaction monitoring (MRM), and quantification and confirmation were performed by MRM of the protonated precursor molecular ions $[\mathrm{M}+\mathrm{H}]^{+}$and the related product ions for each compound by the previously reported method [45]. These MRM transitions and optimal MS/MS parameters are shown in Table S3.

Using this analysis system, calibration curves for nicotine and cotinine were linear in the range of $5-1000 \mathrm{pg} \mathrm{mL}^{-1}$ by comparing peak height ratios with each stable isotopelabeled IS, and detection limits (signal to noise ratio of 3) were 0.45 and $0.13 \mathrm{pg} \mathrm{mL}^{-1}$, respectively. Validation data of this method are shown in Table S1.

\subsection{Preparation and Analysis of Hair Samples and Lifestyle Questionnaires}

Hair samples were provided by 110 healthy Japanese non-smoker volunteers (30 men and 80 women, aged 18-68). All volunteers gave informed consent in writing to the experimental protocol approved by the ethics committee of Shujitsu University (approval code 147; 13 October 2017). The collected hair samples were stored in amber glass desiccator at room temperature until use.

Approximately $10 \mathrm{mg}$ of hair was collected from the back of each subject's head, washed three times with $1 \mathrm{~mL}$ dichloromethane by sonication to remove external nicotine and cotinine from the hair surface, and stored in an amber glass desiccator at room temperature until use. About 1-2 mg of dried hair cut into small pieces with scissors was weighed into a $7 \mathrm{~mL}$ screw-cap vial, to which $1.0 \mathrm{~mL}$ of distilled water and $4 \mu \mathrm{L}$ of the mixed IS solution containing $200 \mathrm{pg}$ of nicotine- $\mathrm{d}_{3}$ and cotinine- $\mathrm{d}_{3}$ were added, and the vial was heated and extracted at $80^{\circ} \mathrm{C}$ for $30 \mathrm{~min}$ with the cap. An aliquot of the extract was transferred to a 2.0-mL autosampler vial and made up to $1 \mathrm{~mL}$ with distilled water, and then used for in-tube SPME/LC-MS/MS analysis. The contents of nicotine and cotinine were calculated as $\mathrm{ng} \mathrm{mg}^{-1}$ and $\mathrm{pg} \mathrm{mg}^{-1}$ hair, respectively, using calibration curves of each compound. 
For all non-smoker subjects, the frequencies of lifestyle behaviors, such as passive smoking, food intake and cooking method were self-reported by questionnaire. Significant differences in the contents of nicotine and cotinine in the hair samples of non-smokers between the scores of these items were analyzed by Student's $t$-test.

\section{Conclusions}

We measured hair nicotine and cotinine levels using an on-line in-tube SPME/LCMS/MS method in 110 Japanese non-smokers who completed to a self-reported lifestyle questionnaire and assessed the effects of environment and lifestyle on these levels. Hair nicotine and cotinine levels were significantly higher in people who were highly aware of passive smoking, and the risk of passive smoking was found to be influenced by the type of food intake and the dietary environment. Nicotine and cotinine in hair are useful biomarkers for assessing the effects of passive smoking due to long-term exposure to environmental tobacco smoke, and our method can analyze the actual exposure with or without awareness of passive smoking. The results of this study show that improving eating habits and lifestyle in various environments are important to avoid the risk of passive smoking.

Supplementary Materials: The following are available online: Figure S1: Outline of on-line in-tube SPME LC-MS/MS system; Table S1: Linearity, sensitivity and precisions of nicotine and cotinine by in-tube SPME LC-MS/MS; Table S2: Program for the in-tube SPME process; Table S3: MRM transitions and setting parameters for nicotine, cotinine and their stable isotope-labeled compounds.

Author Contributions: Conceptualization, H.K.; methodology, H.K., S.K. and M.M.; software, H.K.; validation, H.K., S.K. and M.M.; formal analysis, H.K., S.K. and M.M.; investigation, H.K., S.K. and M.M.; resources, H.K.; data curation, H.K.; writing—original draft preparation, H.K.; writingreview and editing, H.K.; visualization, H.K.; supervision, H.K.; project administration, H.K.; funding acquisition, H.K. All authors have read and agreed to the published version of the manuscript.

Funding: This research was funded by Smoking Research Foundation.

Institutional Review Board Statement: The study was conducted according to the guidelines of the Declaration of Helsinki, and approved by the ethics committee of Shujitsu University (approval code 147; 13 October 2017).

Informed Consent Statement: Informed consent was obtained from all subjects involved in the study.

Data Availability Statement: The data presented in this study are available on request from the corresponding author.

Conflicts of Interest: The authors declare no conflict of interest.

Sample Availability: Samples of the compounds are not available from the authors.

\section{References}

1. Hu, Q.; Hou, H. (Eds.) Tobacco Smoke Exposure Biomarkers; CRC Press, Taylor \& Francis Group: Boca Raton, FL, USA, 2015.

2. Talhout, R.; Schulz, T.; Florek, E.; van Benthem, J.; Wester, P.; Opperhuizen, A. Hazardous compounds in tobacco smoke. Int. J. Environ. Res. Public Health 2011, 8, 613-628. [CrossRef] [PubMed]

3. Mattes, W.; Yang, X.; Orr, M.S.; Richter, P.; Mendrick, D.L. Biomarkers of tobacco smoke exposure. Adv. Clin. Chem. 2014, 67, 1-45. [CrossRef] [PubMed]

4. World Health Organization (WHO). Tobacco Fact Sheet. 2020. Available online: https:/ /www.who.int/news-room/fact-sheets/ detail/tobacco (accessed on 1 October 2021).

5. Öberg, M.; Jaakkola, M.S.; Woodward, A.; Peruga, A.; Prüss-Ustün, A. Worldwide burden of disease from exposure to second-hand smoke: A retrospective analysis of data from 192 countries. Lancet 2011, 377, 139-146. [CrossRef]

6. Torres, S.; Merino, C.; Paton, B.; Correig, X.; Ramírez, N. Biomarkers of exposure to secondhand and thirdhand tobacco smoke: Recent advances and future perspectives. Int. J. Environ. Res. Public Health 2018, 15, 2693. [CrossRef]

7. Hori, M.; Tanaka, H.; Wakai, K.; Sasazuki, S.; Katanoda, K. Secondhand smoke exposure and risk of lung cancer in Japan: A systematic review and meta-analysis of epidemiologic studies. Jap. J. Clin. Oncol. 2016, 46, 942-951. [CrossRef]

8. Kurahashi, N.; Inoue, M.; Liu, Y.; Iwasaki, M.; Sasazuki, S.; Sobue, T.; Tsugane, S. Passive smoking and lung cancer in Japanese non-smoking women: A prospective study. Int. J. Cancer 2008, 122, 653-657. [CrossRef] 
9. $\mathrm{Ni}, \mathrm{X} . ; \mathrm{Xu}, \mathrm{N}$; Wang, Q. Meta-analysis and systematic review in environmental tobacco smoke risk of female lung cancer by research type. Int. J. Environ. Res. Public Health 2018, 15, 1348. [CrossRef]

10. Cao, S.; Yang, C.; Gan, Y.; Lu, Z. The health effects of passive smoking: An overview of systematic reviews based on observational epidemiological evidence. PLoS ONE 2015, 10, e0139907. [CrossRef]

11. Macacu, A.; Autier, P.; Boniol, M.; Boyle, P. Active and passive smoking and risk of breast cancer: A meta-analysis. Breast Cancer Res. Treat. 2015, 154, 213-224. [CrossRef]

12. Lee, P.N.; Hamling, J.S. Environmental tobacco smoke exposure and risk of breast cancer in nonsmoking women. An updated review and meta-analysis. Inhal. Toxicol. 2016, 28, 431-454. [CrossRef]

13. Lee, P.N.; Thornton, A.J.; Hamling, J.S. Epidemiological evidence on environmental tobacco smoke and cancers other than lung or breast. Regul. Toxicol. Pharmacol. 2016, 80, 134-163. [CrossRef]

14. Sheng, L.; Tu, J.W.; Tian, J.H.; Chen, H.J.; Pan, C.L.; Zhou, R.Z. A meta-analysis of the relationship between environmental tobacco smoke and lung cancer risk of nonsmoker in China. Medicine 2018, 97, e11389. [CrossRef]

15. De Groot, P.M.; Wu, C.C.; Carter, B.W.; Munden, R.F. The epidemiology of lung cancer. Transl. Lung Cancer Res. 2018, 7, 220-233. [CrossRef]

16. Law, M.R.; Wald, N.J. Environmental tobacco smoke and ischemic heart disease. Prog. Cardiovasc. Dis. 2003, 46, 31-38. [CrossRef]

17. Ahijevych, K.; Wewers, M.E. Passive smoking and vascular disease. Cardiovasc. Nurs. 2003, 18, 69-74. [CrossRef]

18. Dunbar, A.; Gotsis, W.; Frishman, W. Second-hand tobacco smoke and cardiovascular disease risk: An epidemiological review. Cardiol. Rev. 2013, 21, 94-100. [CrossRef]

19. Messner, B.; Bernhard, D. Smoking and cardiovascular disease: Mechanisms of endothelial dysfunction and early atherogenesis. Arterioscler Thromb. Vasc. Biol. 2014, 34, 509-515. [CrossRef]

20. DiGiacomo, S.I.; Jazayeri, M.A.; Barua, R.S.; Ambrose, J.A. Environmental tobacco smoke and cardiovascular disease. Int. J. Environ. Res. Public Health 2018, 16, 96. [CrossRef]

21. Pistilli, M.; Howard, V.J.; Safford, M.M.; Lee, B.K.; Lovasi, G.S.; Cushman, M.; Malek, A.M.; McClure, L.A. Association of secondhand tobacco smoke exposure during childhood on adult cardiovascular disease risk among never-smokers. Ann. Epidemiol. 2019, 32, 28-34. [CrossRef]

22. Khoramdad, M.; Vahedian-Azimi, A.; Karimi, L.; Rahimi-Bashar, F.; Amini, H.; Sahebkar, A. Association between passive smoking and cardiovascular disease: A systematic review and meta-analysis. IUBMB Life 2020, 72, 677-686. [CrossRef]

23. Akpa, O.M.; Okekunle, A.P.; Asowata, J.O.; Adedokun, B. Passive smoking exposure and the risk of hypertension among non-smoking adults: The 2015-2016 NHANES data. Clin. Hypertens. 2021, 27, 1-10. [CrossRef]

24. Hayashino, Y.; Fukuhara, S.; Okamura, T.; Yamato, H.; Tanaka, H.; Tanaka, T.; Kadowaki, T.; Ueshima, H. A prospective study of passive smoking and risk of diabetes in a cohort of workers. Diabetes Care 2008, 31, 732-734. [CrossRef]

25. Wei, X.; Meng, E.; Yu, S. A meta-analysis of passive smoking and risk of developing type 2 diabetes mellitus. Diabetes Res. Clin. Prac. 2015, 107, 9-14. [CrossRef]

26. Weitzman, M.; Cook, S.R.; Auinger, P.; Florin, T.A.; Daniels, S.; Nguyen, M.; Winickoff, J.P. Tobacco smoke exposure is associated with the metabolic syndrome in adolescents. Circulation 2005, 112, 862-869. [CrossRef]

27. Hamer, M.; Stamatakis, E.; Batty, G.D. Objectively assessed secondhand smoke exposure and mental health in adults: Crosssectional and prospective evidence from the Scottish Health Survey. Arch. Gen. Psychiatry 2010, 67, 850-855. [CrossRef]

28. Chen, R. Association of environmental tobacco smoke with dementia and Alzheimer's disease among never smokers. Alzheimers Dement. 2012, 8, 590-595. [CrossRef]

29. Bamoya, J.; Navas-Acien, A. Protecting the world from secondhand tobacco smoke exposure: Where do we stand and where do we go from here? Nicotine Tob. Res. 2013, 15, 789-804. [CrossRef]

30. Al-Sayed, E.M.; Ibrahim, K.S. Second-hand tobacco smoke and children. Toxicol. Ind. Health 2014, 30, 635-644. [CrossRef]

31. Leonardi-Bee, J.; Smyth, A.; Britton, J.; Coleman, T. Environmental tobacco smoke and fetal health: Systematic review and meta-analysis. Arch. Dis. Child Fetal Neonatal. Ed. 2008, 93, F351-F361. [CrossRef]

32. Abraham, M.; Alramadhan, S.; Iniguez, C.; Duijts, L.; Jaddoe, V.W.; den Dekker, H.T.; Crozier, S.; Godfrey, K.M.; Hindmarsh, P.; Vik, T.; et al. A systematic review of maternal smoking during pregnancy and fetal measurements with meta-analysis. PLoS ONE 2017, 12, e0170946. [CrossRef]

33. Anderson, H.R.; Cook, D.G. Passive smoking and sudden infant death syndrome: Review of the epidemiological evidence. Thorax 1997, 52, 1003-1009. [CrossRef] [PubMed]

34. Adgent, M.A. Environmental tobacco smoke and sudden infant death syndrome: A review. Birth Defects Res. B Dev. Reprod. Toxicol. 2006, 77, 69-85. [CrossRef] [PubMed]

35. Gould, G.S.; Havard, A.; Lim, L.L.; Kumar, R. Exposure to tobacco, environmental tobacco smoke and nicotine in pregnancy: A pragmatic overview of reviews of maternal and child outcomes, effectiveness of interventions and barriers and facilitators to quitting. Int. J. Environ. Res. Public Health 2020, 17, 2034. [CrossRef] [PubMed]

36. Braun, M.; Klingelhöfer, D.M.; Oremek, G.M.; Quarcoo, D.; David, A.; Groneberg, D.A. Influence of second-hand smoke and prenatal tobacco smoke exposure on biomarkers, genetics and physiological processes in children-an overview in research insights of the last few years. Int. J. Environ. Res. Public Health 2020, 17, 3212. [CrossRef] [PubMed]

37. Avila-Tang, E.; Al-Delaimy, W.K.; Ashley, D.L.; Benowitz, N.; Bernert, J.T.; Kim, S.; Samet, J.M.; Hecht, S.S. Assessing secondhand smoke using biological markers. Tob. Control. 2013, 22, 164-171. [CrossRef] 
38. Boumba, V.A.; Ziavrou, K.S.; Vougiouklakis, T. Hair as a biological indicator of drug use, drug abuse or chronic expo-sure to environmental toxicants. Int. J. Toxicol. 2006, 25, 143-163. [CrossRef]

39. Schramm, K.W. Hair-biomonitoring of organic pollutants. Chemosphere 2008, 72, 1103-1111. [CrossRef]

40. Al-Delaimy, W.K. Hair as a biomarker for exposure to tobacco smoke. Tob. Control. 2002, 11, 176-182. [CrossRef]

41. Vogliardi, S.; Tucci, M.; Stocchero, G.; Ferrara, S.D.; Favretto, D. Sample preparation methods for determination of drugs of abuse in hair samples: A review. Anal. Chim. Acta 2015, 857, 1-27. [CrossRef]

42. Pérez-Ortuño, R.; Martínez-Sánchez, J.M.; Fu, M.; Fernández, E.; Pascual, J.A. Evaluation of tobacco specific nitrosamines exposure by quantification of 4-(methylnitrosamino)-1-(3-pyridyl)-1-butanone (NNK) in human hair of non-smokers. Sci. Rep. 2016, 6, 25043. [CrossRef]

43. Benowitz, N.L.; Hukkanen, J.; Jacob, P. Nicotine chemistry, metabolism, kinetics and biomarkers. Handb. Exp. Pharmacol. 2009, 192, 29-60. [CrossRef]

44. Kataoka, H.; Inoue, R.; Yagi, K.; Saito, K. Determination of nicotine, cotinine, and related alkaloids in human urine and saliva by automated in-tube solid-phase microextraction coupled with liquid chromatography-mass spectrometry. J. Pharm. Biomed. Anal. 2009, 49, 108-114. [CrossRef]

45. Inukai, T.; Kaji, S.; Kataoka, H. Analysis of nicotine and cotinine in hair by on-line in-tube solid-phase microextraction coupled with liquid chromatography-tandem mass spectrometry as biomarkers of exposure to tobacco smoke. J. Pharm. Biomed. Anal. 2018, 156, 272-277. [CrossRef]

46. Ishizaki, A.; Kataoka, H. In-tube solid-phase microextraction coupled to liquid chromatography-tandem mass spectrometry for the determination of tobacco-specific nitrosamines in hair samples. Molecules 2021, 26, 2056. [CrossRef]

47. Kataoka, H. In-tube solid-phase microextraction: Current trends and future perspectives. J. Chromatogr. A 2021, $1636,461787$. [CrossRef]

48. Yamamoto, Y.; Ishizaki, A.; Kataoka, H. Biomonitoring method for the determination of polycyclic aromatic hydrocarbons in hair by online in-tube solid-phase microextraction coupled with high performance liquid chromatography and fluorescence detection. J. Chromatogr. B 2015, 1000, 187-191. [CrossRef]

49. Kataoka, H.; Ehara, K.; Yasuhara, R.; Saito, K. Simultaneous determination of testosterone, cortisol and dehydroepiandrosterone in saliva by stable isotope dilution on-line in-tube solid-phase microextraction coupled with liquid chromatography-tandem mass spectrometry. Anal. Bioanal. Chem. 2013, 405, 331-340. [CrossRef]

50. Balcerek, M.; Matławska, I. Preventive role of curcumin in lung cancer. Prz. Lek. 2005, 62, 1180-1181. 